\title{
DANINHAS OCORRENTES NA ZONA SERRANA DO ESTADO DO RIO DE JANEIRO - MUNICÍPIO DE PETRÓPOLIS - 1
}

\author{
Luciana Mautone' \\ Mítzi Brandrão ${ }^{2}$ \\ Elsie Franklin Guimarães ${ }^{3}$ \\ João Rodrigues Miguel $^{4}$
}

RESUMO - São apresentadas as plantas daninhas ocorrentes no Município de Petrópolis, recolhidas em 14 estaçōes de coleta, das 60 programadas. Foram cadastradas cerca de 152 espécies, pertencentes a 104 gêneros e 46 famílias.

Palavras-chave: Plantas daninhas do Estado do Rio de Janeiro.

ABSTRACT - A list of weedy species collected in Petropolis (Rio de Janeiro State) is presented comprising 152 species of 104 genera belonging to 46 families.

\section{Introdução}

O estudo das plantas daninhas ocorrentes no Estado do Rio de Janeiro, sempre foi objeto de interesse, hoje em desenvolvimento, sob a forma de Projeto: "Plantas daninhas do Estado do Rio de Janeiro", que nesta etapa, enfatiza o levantamento daquelas do município de Petrópolis.

Para o estudo das plantas consideradas daninhas do município em questão foram selecionadas 60 estações de coleta com abrangência de todos os bairros.

O trabalho sob enfoque, objetiva além do conhecimento destas plantas, a verificação de novas ocorrências para o Estado.

1 - Pesquisadora em Ciências Exatas e da Natureza - IBAMA/JBRJ; Rua Jardim Botânico no 1008 - CEP 22460 - Rio de Janeiro-RJ.

2 - Pesquisadora da Empresa de Pesquisa Agropecuária de Minas Gerais - EPAMIG, Rua da Bahia, 360, 4 andar - CEP 30.160, B elo Horizonte. Bolsista do CNPq.

3 - Pesquisadora em Giências Exatas e da Natureza - IBAMA/JBRJ. Bolsista do CNPq.

4 - Biólogo. 


\section{Breve histórico sobre Petrópolis}

Em 1720, Bernardo Soares de Proença resolveu abrir uma variante do caminho Rio-Minas, subindo e descendo a Serra da Estrela, e assim, o território de Petrópolis começou a ser desbravado, (Ferrez, 1970).

Em 1822, D. Pedro I passou pelo local em missão política e, mais tarde, por motivo de doença de sua filha, adquiriu o Córrego Seco.

Com a chegada de uma grande leva de imigrantes alemães, em 29 de junho de 1845, grande impulso foi dado à Petrópolis, que então, foi elevada à categoria de cidade (1857), portanto, desmembrada da Vila da Estrela, passando a município em 1859 (Ferrez, 1970); (Rahal, 1984).

O palácio construído pelo Major Julio Frederico Koeler, natural de Mayen na Prússia, hoje Museu Imperial, apresenta em sua fachada motivos gregos de decoração, colunas jônicas e coríntias, e ao alto da frontaria principal, as armas imperiais, os dragões dos Braganças, o cetro e a mão da Justiça, tornando-se a residência predileta de D. Pedro II.

Como as terras eram impróprias à agricultura, foram aproveitadas para estabelecimentos industriais e comerciais, tendo-se tornado mais tarde uma cidade de veraneio, procurada pela realeza e corpo diplomático.

Em 1903, com a mudança do Governo do Estado, multiplicaram-se os hotéis, educandários, estabelecimentos oficiais e comerciais, construindo-se os Palácios do Estado e da República, além, de numerosas sedes de Embaixadas.

Em 1928, com a abertura da estrada de rodagem e pouco depois com a sua pavimentação, e a reconstrução de rodovia para Minas Gerais, tornou-se ponto obrigatório de passagem para o interior do país, desfrutando hoje, de posição invejável entre as demais cidades do Estado.

Sua área é de $1.080 \mathrm{~km}^{2}$ com cerca de 241.884 habitantes, situando-se a $70 \mathrm{~km}$ do Rio de Janeiro, possuindo clima ameno, com temperaturas máximas de $24^{\circ} \mathrm{C}$, apresentando $810 \mathrm{~m}$ de altitude. O município localiza-se na zona fisiográfica do Alto da Serra, limitando-se com Duque de Caxias, Magé, Miguel Pereira, Paraíba do Sul, Sapucaia, Teresópolis e Vassouras.

O município apresenta relevo montanhoso, encontrando-se como principais acidentes orográficos, as Serras dos Órgãos e da Estrela.

O revestimento florístico é caracterizado pelas exuberantes matas das encostas e por campos de várzeas encaixados.

Tornou-se por Decreto de 25.03.81 a cidade império, única na América Latina. 


\section{Material e Métodos}

Para o presente trabalho foi realizada uma primeira excursão para demarcação das estações de coletas (60 estações).

Para a presente etapa foram selecionadas 14 estaçōes: (1) Bonfim, (2) Carangola, (3) Amoedo, (4) Nogueira, (5) Quarteirão, (6) Duchas, (7) Centro, (8) Correas, (9) Retiro, (10) Barão do Rio Branco, (11) Museu/Prefeitura, (12) Bingen, (13) Moinho Preto (14) Mosela.

$\mathrm{Na}$ execução do trabalho foram realizadas mais três excursões, durante o período de 2 anos, utilizando-se os procedimentos seguintes:

a - excursões para as estações escolhidas

b - coleta de material botânico

c-etiquetagem e prensagem

d - observaçöes de campo

e - posteriormente, as exsicatas foram identificadas, de acordo com os métodos tradicionais utilizados para tal finalidade, após o que, foram montadas, etiquetadas, registradas e depositadas no Herbário do Jardim Botânico do Rio de Janeiro.

f - foi confeccionada a tabela 1 e a listagem das plantas coletadas.

Quando do estudo e identificação do material coletado foram consultados os yutores: Barreiros, 1970; Barroso, 1952 a e b, 1956, 1959, 1962/1965; Benjamin, 1959; Brandão, et al, 1982, 1985, 1989; Brandão, 1985; Carvalho, 1959; Falcão, 1966; Falcão et al, 1979; Ferreira \& Laca-Buendia, 1978; Kuhlmann et al., 1947; Leitão Filho et al., 1972, 1972, 1975; Marinis, 1970; Monteiro, 1933; Monteiro Filho, 1936, 1954, 1956; Paluma, 1984; Pereira \& Brandão, 1988; Pereira \& Agarez, 1974; Sarahyba \& Lemos, 1984; Siqueira \& Guimarães, 1984 e Teixeira, 1956.

\section{Resultados}

O levantamento das plantas daninhas do Município de Petrópolis realizado até o presente momento, em apenas 14 estações de coleta (das 60 estações), revelou que as famílias Compositae, Gramineae, Amaranthaceae concorrem com um maior número de espécies, respectivamente 31,9 e 8.

Synedrellopsis grisebach Hieron \& Kuntze, embora mencionada para o Estado do Rio de Janeiro (Lorenzi, 1982), não se encontrava inclusa na listagem para o Estado.

Bauhinia microstachya (Raddi) Macbr. Sycios polycanthos Cogn., Cleobulia multiflora Martex., Piper mollicomum Kunth, Peperonia pellucida (L), HBK, Polygala fimbriata Benn., Solanum violaefolium Schott apresentavam comportamento como invasoras, no município em questão, embora não consideradas como tais, pela literatura pertinente, acima relacionada. 
Entre as espécies mencionadas ocorrem plantas tidas como ornamentais evadidas dos jardins, no momento encontrando-se na senda das plantas invasoras, como Tropaelum majus L.e Impatiens balsamina $L$.

Foram cadastradas 152 espécies pertencentes a 104 gêneros, compondo 46 famílias.

As espécies encontradas para as 14 estações são apresentadas na Tabela

Tabela 1 - Lista de famílias encontradas nas 14 estações de coleta, mostrando o número de gêneros e espécies.

\begin{tabular}{|c|c|c|}
\hline Família & Gênero & Espécie \\
\hline Acanthaceae & 1 & 1 \\
\hline Amaranthaceae & 2 & 8 \\
\hline Alismataceae & 2 & 2 \\
\hline Asclepiadaceae & 1 & 1 \\
\hline Balsaminaceae & 1 & 1 \\
\hline Bignoniaceae & 1 & 1 \\
\hline Caesalpinaceae & 2 & 4 \\
\hline Capparidaceae & 1 & 2 \\
\hline Caryophyllaceae & 2 & 2 \\
\hline Chenopodiaceae & 1 & 2 \\
\hline Commelinaceae & 2 & 3 \\
\hline Compositae & 24 & 31 \\
\hline Convolvulaceae & 3 & 6 \\
\hline Cucurbitaceae & 4 & 4 \\
\hline Cuscutaceae & 1 & 1 \\
\hline Cruciferae & 3 & 5 \\
\hline Cyperaceae & 1 & 6 \\
\hline Euphorbiaceae & 4 & 9 \\
\hline Fabaceae & 4 & 6 \\
\hline Gramineae & 9 & 9 \\
\hline Haloragaceae & 1 & 1 \\
\hline Hypoxidaceae & 1 & 1 \\
\hline Labiatae & 4 & 5 \\
\hline Lythraceae & 1 & 2 \\
\hline Malvaceae & 2 & 4 \\
\hline Mimosaceae & 1 & 1 \\
\hline Molluginaceae & 1 & 1 \\
\hline Moraceae & 1 & 1 \\
\hline Nyctaginaceae & 1 & 1 \\
\hline Oxalidaceae & 1 & 3 \\
\hline Phytollacaceae & 1 & 1 \\
\hline Piperaceae & 2 & 2 \\
\hline Plantaginaceae & 1 & 2 \\
\hline Polygalaceae & 1 & 2 \\
\hline Polygonaceae & 2 & 6 \\
\hline Portulacaceae & 2 & 2 \\
\hline
\end{tabular}




\begin{tabular}{lcc}
\hline \multicolumn{1}{c}{ Familia } & Gênero & Espécie \\
\hline Polypodiaceae & 1 & 1 \\
Rosaceae & 1 & 1 \\
Rubiaceae & 5 & 7 \\
Scrophulariaceae & 1 & 1 \\
Solanaceae & 3 & 7 \\
Tiliaceae & 1 & 2 \\
Tropeolaceae & 1 & 1 \\
Turneraceae & 1 & 1 \\
Umbelliferae & 2 & 2 \\
Urticaceae & 1 & 1 \\
Verbenaceae & 2 & 3 \\
Total & 104 & 152 \\
\hline
\end{tabular}




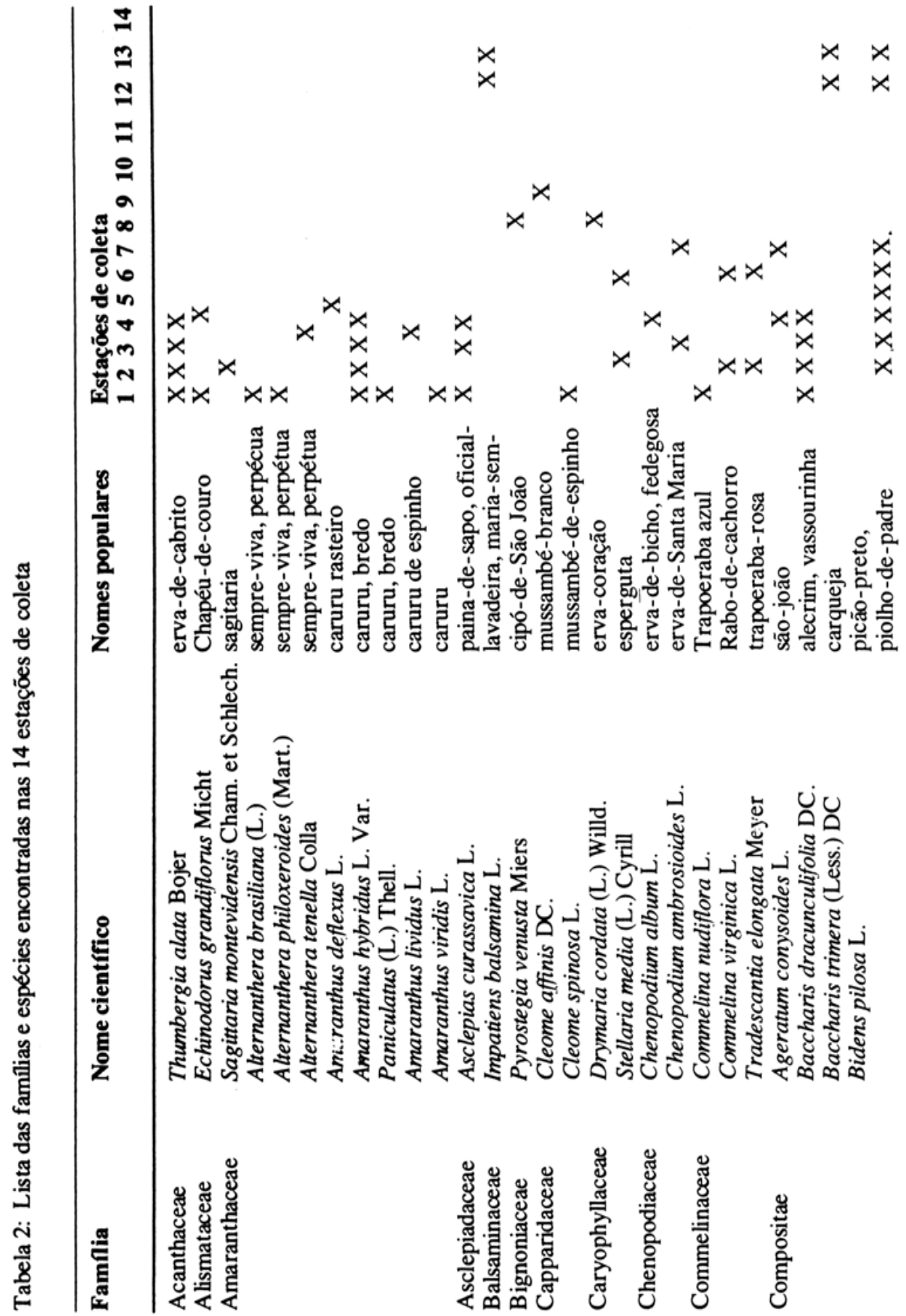




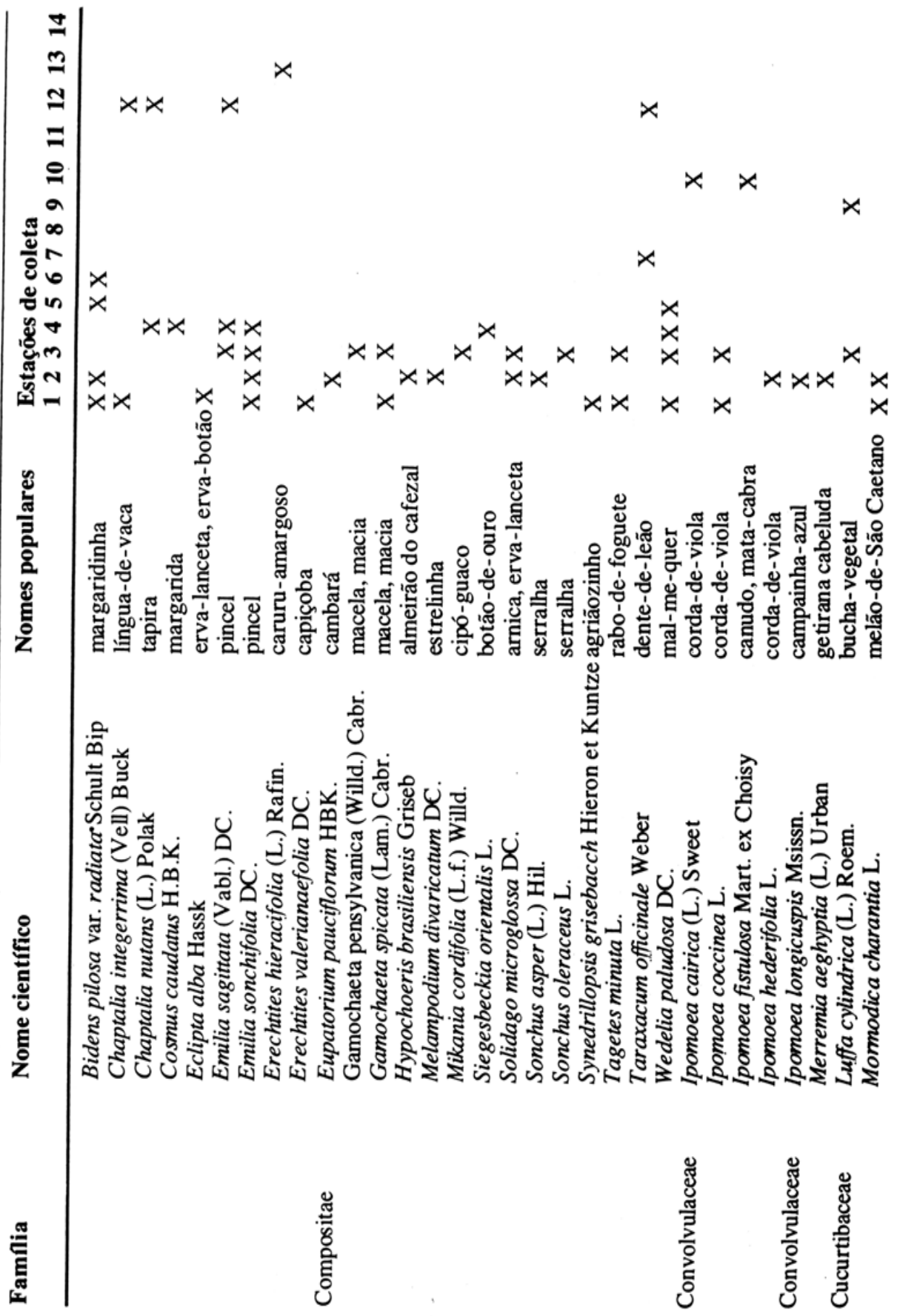




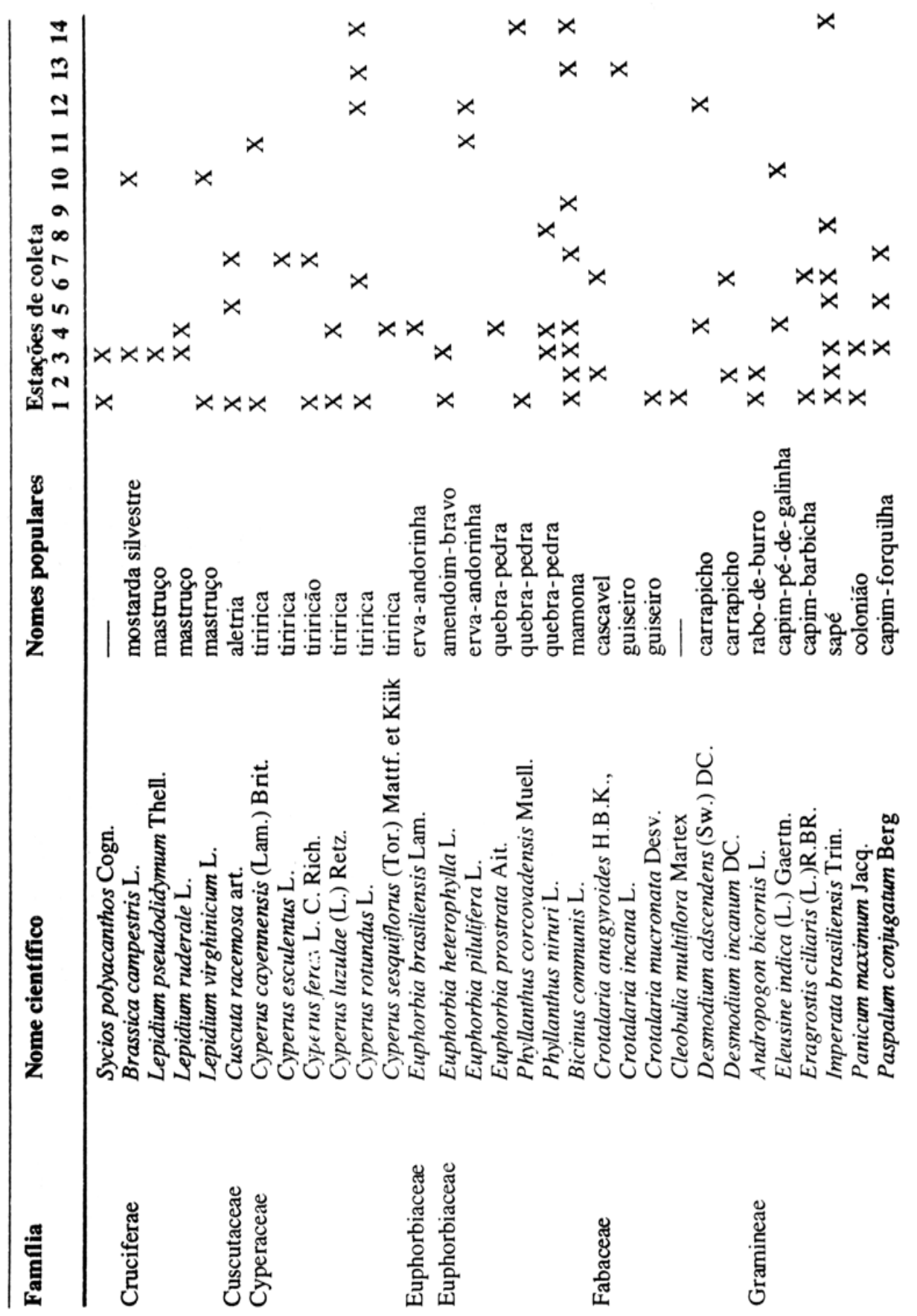




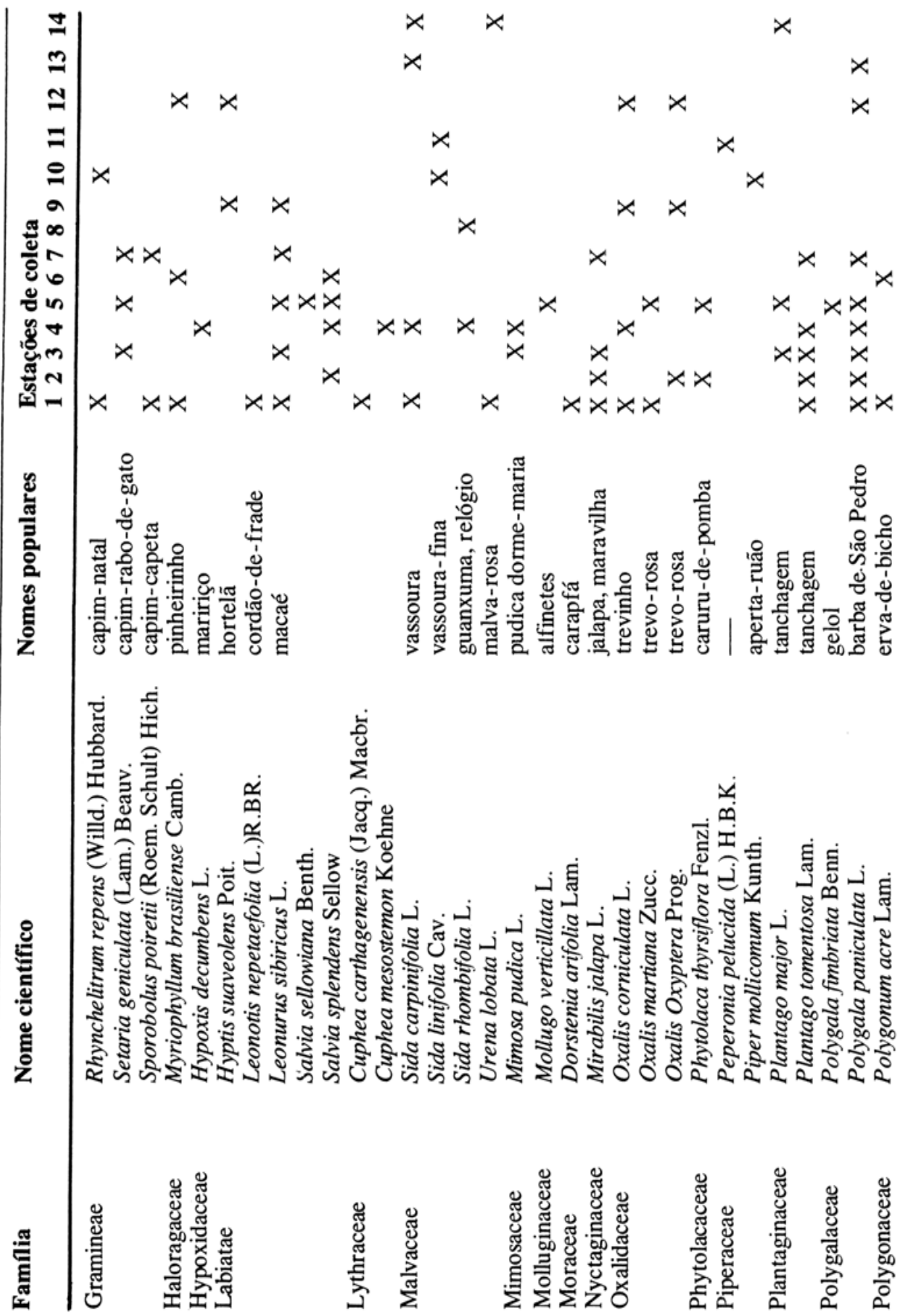




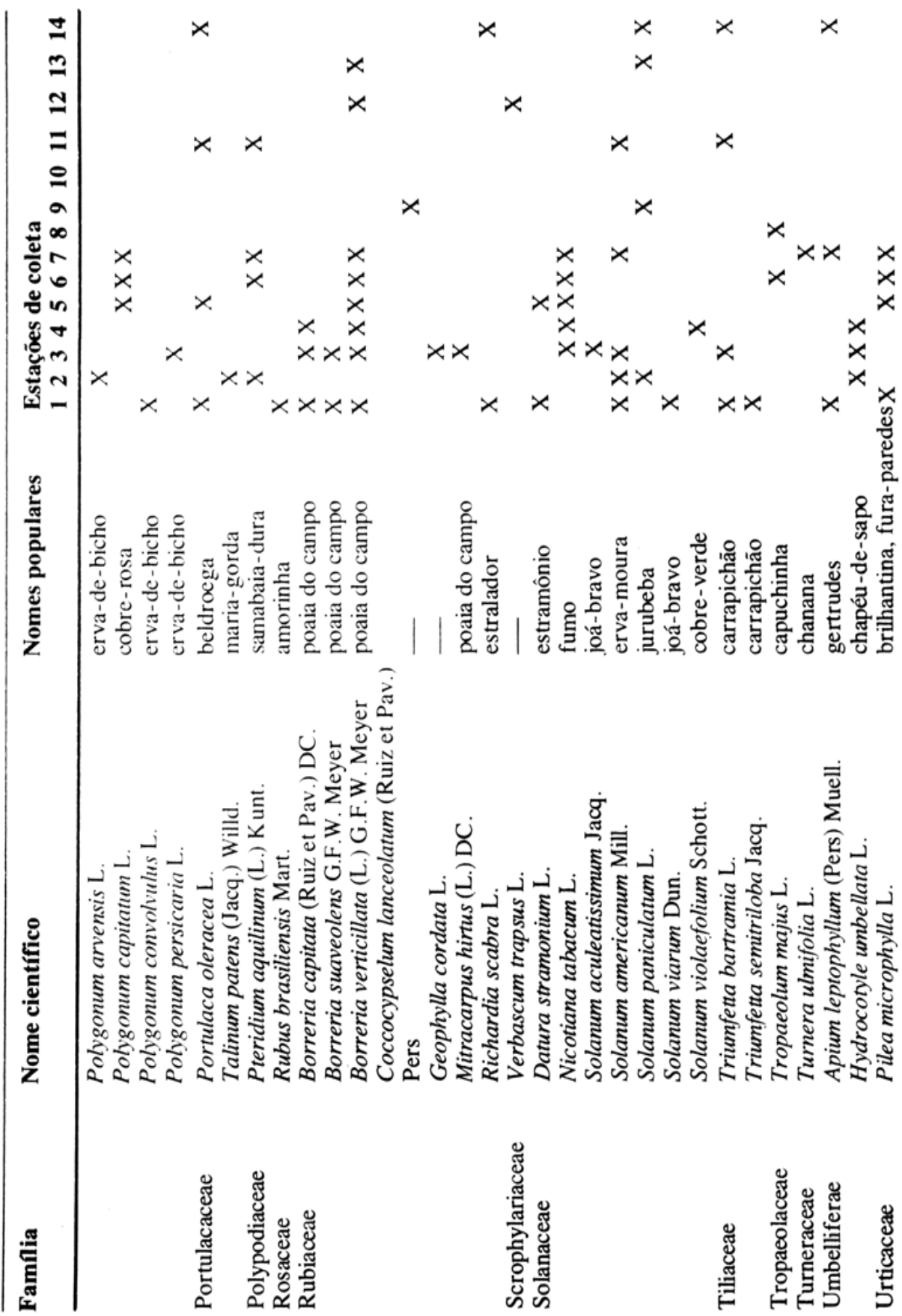




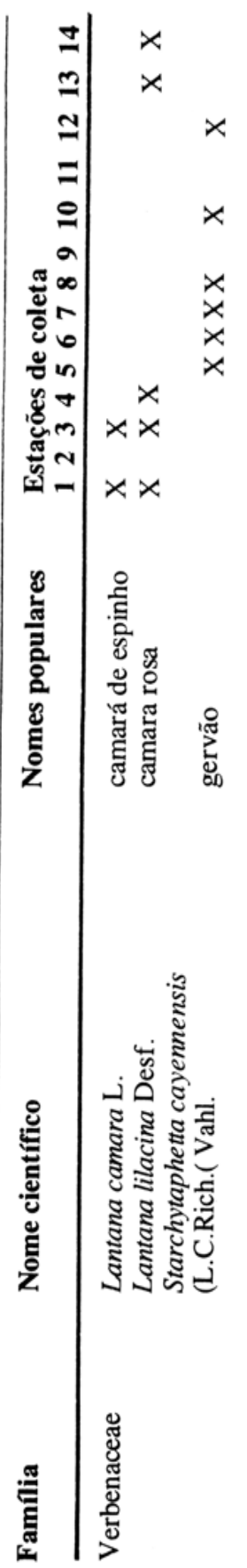




\section{Referências bibliografficas}

BARREIROS, H. de S. 1970. Espécies de monocotiledoneas que ocorrem no Estado da Guanabara - 1. Cyperaceae (Glumiflorae). Atas da Soc. Biol. Rio de Janeiro. 13(5-6):68-173.

BARROSO, G.M. 1952. Contribuição ao estudo do gênero Cuphea Adans. Rodriguesia, Rio de Janeiro 15(27):9-108.

BARROSO, G.M. 1952 (b). Scrophulariaceae indígenas e exóticas do Brasil Rodriguesia, Rio de Janeiro, 15(27):109-115.

BARROSO, G.M. 1956, Flora da cidade do Rio de Janeiro (Compositae) Rodriguesia, Rio de Janeiro, 21/22 (33/34):66-147.

BARROSO, G.M. 1959 - Flora da cidade do Rio de Janeiro (Compositae) Rodriguesia, Rio de Janeiro, 24/25 (37/38)69-155.

BARROSO G.M. 1962/1965 - Leguminosas da Guanabara. Arq. do Jardim Botânico Rio de Janeiro, 18:101-177.

BENJAMIM, D.S. - 1959. Rubiaceae da cidade do Rio de Janeiro, 1 Tribo Spermacoeae. Rodriguesia, Rio de Janeiro. 21/22(33/34):241-280.

BRANDÃO, M. 1985. Plantas invasoras de pastagens do município de Cantagalo, RJ. In: Congresso Nacional de Botânica, 36, Curitiba, 1985. Resumos, Curitiba, 1985 , p. 80.

BRANDÃO, M; E.F. GUIMARÃES; L. MAUTONE \& A. MATTOS FILHO. 1989. - Plantas daninhas ocorrentes na área do Parque do Jardim Botânico do Rio de Janeiro, Rodriguesia (no prelo).

BRANDÄO, M; J. P. LACA-BUENDIA; \& N.L. GAVILANES, 1982. Principais plantas daninhas do estado de Minas Gerais. Informe agropecuário. B:lo Horizonte, 8 (87): 18-27.

BRANDÃO, M. \& M.E. PALUMA. 1985 - Plantas daninhas pouco conhecidas ou ainda não mencionadas como ocorrentes em culturas ou pastagens, no estado do Rio de Janeiro. In: Congresso Nacional de Botânica, 36, Curitiba, 1985, Resumos, Curitiba, p. 82. (no prelo).

CARVALHO, L.F. de 1959 - Plantas invasoras de culturas do Estado do Rio de Janeiro. In: Seminário Brasileiro de Herbicidas e Ervas Daninhas 2, Belo Horizonte, 1958. Anais, Rio de Janeiro, Centro Nacional de Ensino e Pesquisas Agronômicas. p. 115-23.

FALCÃO, J.I. 1966 - Convolvulaceae do Estado`da Guanabara, Rodriguesia, Rio de Janeiro, 25(37):141-160.

FALCÃO, W.F.A., \& J.I. FALCÃO. 1979 - Convolvulaceae do Rio de Janeiro, Rodriguesia, Rio de Janeiro, 31(50):7-35.

FERREIRA, M.B. \& J.P. LACA BUENDIA. 1978. Espécies consideradas plantas daninhas em áreas cultivadas no Estado de Minas Gerais. Planta Daninha, Campinas, 1(2):16-26.

KUHLMANN, J. G., P. OCCHIONI \& J.L.A. FALCÃO - 1947. Contribuição ao estudo das plantas ruderais do Brasil. Arq. de Jard. Botanico, 
Rio de Janeiro 7: 43-133.

LEITÃO FILHO, H.F., C. ARANHA \& O. BACCHI. 1972 - Plantas invasoras de culturas do Estado de São Paulo, São Paulo, Hucitec, v.1.

LEITÃO FILHO, H.F. C. ARANHA \& O. BACCHI. 1975. Plantas invasoras de cultura do Estado de São Paulo, São Paulo, Hucitec, v.2.

LORENZI, H. Plantas daninhas do Brasil-terrestres, aquáticas, parasitas, tóxicas e medicinais. Nova Odessa, São Paulo, H. Lorenzi, 1982, 425 p.

MARINIS, G. 1970. Espécies invasoras brasileiras do gênero Alternanthera In: Seminário Brasileiro de Herbicidas e Ervas Daninhas, Botucatu, 1970. Resumos p1-2.

MONTEIRO, H. 1933. As portulacas sob o ponto de vista fitogeográfico, Rio de Janeiro, Min. da Agricultura, Divisão de plantas téxteis, p.4. (publ. no 2).

MONTEIRO FILHO, H. C. 1936. Monografia das Malvaceae brasileiras. Fasc. 1. O gênero Sida. Revisão das espécies brasileiras; chave para as espécies sul-americanas e novidades brasileiras. Rio de Janeiro, $\mathrm{Ma} / \mathrm{Dnpv} / \mathrm{Spt} .49 \mathrm{p}$.

MONTEIRO FILHO, H.C. 1954 - Malvaceae espontâneas e subespontâneas do km 47 (Universidade Rural), Agronomia, 13(1): 49-56.

MONTERIRO FILHO, H.C. 1956 - Malvaceae fluminensis invasoras de culturas. In: Seminário Brasileiro de Herbicidas e Ervas Daninhas. Rio de Janeiro, 1956. Anais. Rio de Janeiro, p 185-194.

PALUMA, M.E. 1984 - Plantas daninhas mun. de Niterói, RJ. Pesagro/Rio Niterói.

PEREIRA, C. 1962/65. Flora do Estado da Guanabara (Labiatae). Arq. do Jardim Botânico, Rio de Janeiro. 18:87-108.

PEREIRA, C.B. \& F.U. AGAREZ. 1974 - Estudo das plantas ruderais do Estado da Guanabara. Leandra, Rio de Janeiro. 3/4 (4/5):67-83, 15 est.

PEREIRA, P.P. \& M. BRANDÃO. 1988 - Lavantamento e identificação de plantas invasoras da cultura do arroz na região norte do estado do Rio de Janeiro. Pesagro/Rio 28. (Boletim técnico; 12).

SARAHYBA, L.S.P. \& F.S.P. LEMOS. 1984. Gramineae do Estado do Rio de Janeiro - Lita de espécies./Atas da Sociedade Botânica do Brasil, 2(16):121-132.

SIQUEIRA, J.C. \& E.F. GUIMARÃES. 1984 - Amaranthaceae do Rio de Janeiro, Rodriguesia, Rio de Janeiro, 36(58):21-40.

TEIXEIRA, L. 1956 - Portulacaceae da cidade do Rio de Janeiro. Rodriguesia 21/22(33/34):299-306. 